\title{
Root Sets of Polynomials and Power Series with Finite Choices of Coefficients
}

\author{
Simon Baker ${ }^{1}$ - Han Yu ${ }^{2}$
}

Received: 21 April 2017 / Revised: 6 June 2017 / Accepted: 20 June 2017 /

Published online: 9 October 2017

(C) The Author(s) 2017. This article is an open access publication

Abstract Given $H \subseteq \mathbb{C}$ two natural objects to study are the set of zeros of polynomials with coefficients in $H$,

$$
\left\{z \in \mathbb{C}: \exists k>0, \exists\left(a_{n}\right) \in H^{k+1}, \sum_{n=0}^{k} a_{n} z^{n}=0\right\}
$$

and the set of zeros of a power series with coefficients in $H$,

$$
\left\{z \in \mathbb{C}: \exists\left(a_{n}\right) \in H^{\mathbb{N}}, \sum_{n=0}^{\infty} a_{n} z^{n}=0\right\} .
$$

In this paper, we consider the case where each element of $H$ has modulus 1 . The main result of this paper states that for any $r \in(1 / 2,1)$, if $H$ is $2 \cos ^{-1}\left(\frac{5-4|r|^{2}}{4}\right)$ dense in $S^{1}$, then the set of zeros of polynomials with coefficients in $H$ is dense in $\left\{z \in \mathbb{C}:|z| \in\left[r, r^{-1}\right]\right\}$, and the set of zeros of power series with coefficients in $H$ contains the annulus $\{z \in \mathbb{C}:|z| \in[r, 1)\}$. These two statements demonstrate

Communicated by Thomas Ransford.

$凶 \quad \mathrm{Han} \mathrm{Yu}$

hy25@st-andrews.ac.uk

Simon Baker

simonbaker412@gmail.com

1 Mathematics Institute, University of Warwick, Coventry CV4 7AL, UK

2 School of Mathematics and Statistics, University of St Andrews, St Andrews KY16 9SS, UK 
quantitatively how the set of polynomial zeros/power series zeros fill out the natural annulus containing them as $H$ becomes progressively more dense.

Keywords Root set $\cdot$ Littlewood polynomials $\cdot$ Unimodular polynomials

Mathematics Subject Classification Primary 30B30; Secondary 30C15 · 11C08

\section{Introduction}

Let $H \subseteq \mathbb{C}$ be a finite set. Given such a $H$ we define the root set of polynomials with coefficients in $H$ to be:

$$
R(H):=\left\{z \in \mathbb{C}: \exists k>0, \exists\left(a_{n}\right) \in H^{k+1}, \sum_{n=0}^{k} a_{n} z^{n}=0\right\}
$$

Similarly, we define the root set of power series with coefficients in $H$ to be

$$
R^{*}(H):=\left\{z \in \mathbb{C}: \exists\left(a_{n}\right) \in H^{\mathbb{N}}, \sum_{n=0}^{\infty} a_{n} z^{n}=0\right\} .
$$

The study of the sets $R(H)$ and $R^{*}(H)$ can be dated back to Littlewood [6] who studied the case where $H=\{-1,1\}$. Since then many related works have appeared, most notable amongst these are the number theoretic results of Beaucoup, Borwein, Boyd and Pinner [3], and Borwein, Erdélyi and Littmann [4], who studied the distribution of roots and multiple roots. Related work also appeared in Bousch [5], where it was shown that $R(\{-1,1\})$ is dense in $\left\{z:|z|^{4} \in[1 / 2,2]\right\}$. In Shmerkin and Solomyak [8] some measure theoretic and topological properties of $R(\{-1,0,1\})$ are studied in detail.

In what follows, we will adopt the following notational conventions:

$$
S^{r}:=\{z \in \mathbb{C}:|z|=r\}, B(z, r):=\left\{z^{\prime} \in \mathbb{C}:\left|z^{\prime}-z\right|<r\right\},
$$

and given some interval $I$ in $\mathbb{R}$ let

$$
A_{I}:=\{z \in \mathbb{C}:|z| \in I\} .
$$

In this paper, we focus on the case where $H$ is a subset of the unit circle $S^{1}$. Under this assumption it is straightforward to show that

$$
R(H) \subseteq A_{[1 / 2,2]} \text { and } R^{*}(H) \subseteq A_{[1 / 2,1)}
$$

Intuitively, one might expect that if we allowed $H$ to become a progressively more dense subset of $S^{1}$, then $R(H)$ and $R^{*}(H)$ would begin to fill out their respective annuli. The main result of this paper shows that this intuition is correct. 
Before stating this result we need to define a metric on $S^{1}$ to properly quantify the density of $H$. Given $e^{i \theta}, e^{i \theta^{\prime}} \in S^{1}$ let $d\left(e^{i \theta}, e^{i \theta^{\prime}}\right)=\min \left\{\left|\theta-\theta^{\prime}\right|,\left|2 \pi-\left(\theta-\theta^{\prime}\right)\right|\right\}$. This metric measures the interior angle of the sector of $S^{1}$ determined by the two radii $e^{i \theta}$ and $e^{i \theta^{\prime}}$.

Theorem 1.1 Fix $r \in(1 / 2,1)$. Suppose $H \subseteq S^{1}$ is $2 \cos ^{-1}\left(\frac{5-4 r^{2}}{4}\right)$-dense. Then $A_{[r, 1)} \subseteq R^{*}(H)$ and $R(H)$ is dense in $A_{\left[r, r^{-1}\right]}$.

The sets $R(H)$ and $R^{*}(H)$ are related by the following formula.

Proposition 1.2 Let $H \subseteq \mathbb{C}$ be any finite set, then the following relations hold:

$$
R(H)=\frac{1}{R(H)},
$$

and

$$
\overline{R(H)} \cap B(0,1)=R^{*}(H) \cap B(0,1) .
$$

In the statement of Proposition 1.2, $\bar{A}$ denotes the closure of a set $A$, and $\frac{1}{A}$ denotes the set $\left\{z \in \mathbb{C}: z^{-1} \in A\right\}$.

Proof Given $z \in \mathbb{C}$ suppose there is polynomial $P \in H[x]$ such that,

$$
P(z)=\sum_{n=0}^{k} a_{n} z^{n}=0 .
$$

We can construct another polynomial $Q \in H[x]$ such that $Q(1 / z)=0$. Just consider $Q(x)=x^{k} P\left(\frac{1}{x}\right)$ with $k=\operatorname{deg} P$. Therefore, whenever $z \in R(H)$ we also have $1 / z \in R(H)$. This proves our first relation.

Now we shall show that,

$$
\overline{R(H)} \cap B(0,1)=R^{*}(H) \cap B(0,1) .
$$

Without loss of generality we can assume that $H \subseteq\{z:|z| \leq 1\}$. If $z^{*} \in \overline{R(H)} \cap$ $B(0,1)$ then we can find a sequence $\left(z_{i}\right) \in R(H)^{\mathbb{N}}$ with:

$$
z_{i} \rightarrow z^{*}
$$

Moreover, since $z^{*} \in B(0,1)$ there exists a positive number $M$ such that $1<M<\frac{1}{\left|z^{*}\right|}$. Now let us consider any polynomial in $H[x]$

$$
P(x)=\sum_{n=0}^{k} a_{n} x^{n} .
$$


The following result holds for $\left|z_{i}\right| \leq M\left|z^{*}\right|$

$$
\begin{aligned}
\left|P\left(z^{*}\right)-P\left(z_{i}\right)\right| & \leq \sum_{n=0}^{k}\left|a_{n}\right|\left|\left(z^{*}\right)^{n}-z_{i}^{n}\right| \\
& =\sum_{n=0}^{k}\left|a_{n}\right|\left|z^{*}-z_{i}\right|\left|\left(z^{*}\right)^{n-1}+\left(z^{*}\right)^{n-2} z_{i}+\ldots+z_{i}^{n-1}\right| \\
& \leq \sum_{n=0}^{k}\left|a_{n}\right|\left|z^{*}-z_{i}\right| n\left(M\left|z^{*}\right|\right)^{n-1} \\
& \leq\left|z^{*}-z_{i}\right| \sum_{n=0}^{k}\left|a_{n}\right| n\left(M\left|z^{*}\right|\right)^{n-1} .
\end{aligned}
$$

Since $\left|a_{n}\right| \leq 1$ and $M\left|z^{*}\right|<1$ the latter summation can be bounded uniformly with respect to $k$, namely:

$$
\sum_{n=0}^{k}\left|a_{n}\right| n\left(M\left|z^{*}\right|\right)^{n-1} \leq C,
$$

where $C>0$ is a constant that only depends upon $z^{*}$.

Each $z_{i}$ is the root of some polynomial $P_{i} \in H[x]$, in which case by the above, for $i$ sufficiently large we have

$$
\left|P_{i}\left(z^{*}\right)\right|=\left|P_{i}\left(z^{*}\right)-P_{i}\left(z_{i}\right)\right| \leq C\left|z^{*}-z_{i}\right|
$$

For the sequence $\left(P_{i}\right)$ there is either a uniform upper bound for the degrees of the $P_{i}$, or there exists a subsequence along which the degrees tend to infinity. In the first case, there must exist a polynomial $Q \in H[x]$ and a subsequence $\left(P_{i_{j}}\right)$ such that $P_{i_{j}}=Q$ for all $i_{j}$. By (1) we must then have $Q\left(z^{*}\right)=0$. Suppose $\operatorname{deg} Q=L$, then

$$
T(x)=Q(x) \sum_{n=0}^{\infty} x^{n(L+1)}
$$

is a power series with digits in $H$. For this particular power series we clearly have $T\left(z^{*}\right)=0$. Therefore, in the first case we have $z^{*} \in R^{*}(H)$. Now suppose there exists a subsequence $\left(P_{i_{j}}\right)$ such that $\operatorname{deg} P_{i_{j}} \rightarrow \infty$. Via a diagonalisation argument, one can assume without loss of generality that there exists a sequence $\left(a_{n}\right) \in H^{\mathbb{N}}$ and an increasing sequence of natural numbers $\left(l_{n}\right)$, such that for all $i_{j} \geq l_{n}$ the coefficient of the degree $n$ term of $P_{i_{j}}$ is $a_{n}$. In other words, as the $i_{j}$ become sufficiently large the lower order terms of the $P_{i_{j}}$ 's start to coincide. It follows from (1) then that for this sequence $\left(a_{n}\right)$ we must have 


$$
\sum_{n=0}^{\infty} a_{n}\left(z^{*}\right)^{n}=0
$$

Therefore, $z^{*} \in R^{*}(H)$ and $\overline{R(H)} \cap B(0,1) \subseteq R^{*}(H) \cap B(0,1)$.

Now suppose $z^{*} \in R^{*}(H) \cap B(0,1)$. Then there is a sequence $\left(a_{n}\right) \in H^{\mathbb{N}}$ such that

$$
\sum_{n=0}^{\infty} a_{n}\left(z^{*}\right)^{n}=0
$$

This series is absolutely and uniformly convergent in $B(0, c)$ for any $0<c<1$. Since $z^{*} \in B(0,1)$ it is contained in one of these sets for $c$ sufficiently close to 1 . We see that the function:

$$
P(x)=\sum_{n=0}^{\infty} a_{n} x^{n}
$$

is holomorphic on the interior of the unit disc, and therefore, the roots of $P$ must form a discrete set. Since $z^{*}$ belongs to the root set of $P$ there must exist $r>0$ such that

$$
\{z \in \mathbb{C}: P(z)=0\} \cap B\left(z^{*}, r\right)=\left\{z^{*}\right\} .
$$

Now, suppose that $z^{*} \notin \overline{R(H)}$, then there exists a ball $B\left(z^{*}, r^{\prime}\right)$ such that $B\left(z^{*}, r^{\prime}\right) \subseteq$ $B\left(z^{*}, r\right)$ and

$$
R(H) \cap \overline{B\left(z^{*}, r^{\prime}\right)}=\emptyset .
$$

We can then consider the following integral with $P_{N}(x)=\sum_{n=0}^{N} a_{n} x^{n}$

$$
I_{N}=\int_{\partial B\left(z^{*}, r^{\prime}\right)} \frac{P_{N}^{\prime}(x)}{P_{N}(x)} \mathrm{dx} .
$$

By our conditions on $r^{\prime}$ we see that $P_{N}$ has no zeros in $\overline{B\left(z^{*}, r^{\prime}\right)}$ for all $N \in \mathbb{N}$. Therefore, by the argument principle (see [1, p. 152]) we must have $I_{N} \equiv 0$. One can also assume that $r^{\prime}$ is sufficiently small so that $P_{N}$ converges to $P$ absolutely and uniformly. Therefore,

$$
0=\lim _{N \rightarrow \infty} I_{N}=\int_{\partial B\left(z^{*}, r^{\prime}\right)} \frac{P^{\prime}(x)}{P(x)} \mathrm{dx} .
$$

However, it follows from another application of the argument principle, and the fact that $P(x)$ has a single zero in $\overline{B\left(z^{*}, r^{\prime}\right)}$ at $z^{*}$, that the above integral cannot be 0 . This contradiction implies $z^{*} \in \overline{R(H)}$ and our proof is complete. 
It is natural to wonder whether there exists a set $H$ such that the sets $R(H)$ and $R^{*}(H)$ fill up their ambient annuli, that is $A_{[1 / 2,2]}$ and $A_{[1 / 2,1)}$ respectively. In fact such a $H$ cannot exist. For any $H \subseteq S^{1}$ there exists $z \in \mathbb{C}$ with modulus $1 / 2$ and $\delta>0$, such that $R(H) \cap B(z, \delta)=\emptyset$ and $R^{*}(H) \cap B(z, \delta)=\emptyset$. This is because of the following simple reasoning. Since $H$ is a finite set there exists $z \in \mathbb{C}$ such that $|z|=1 / 2$ and

$$
\left|a_{i}+a_{j} z\right|>1 / 2
$$

for all $a_{i}, a_{j} \in H$. Equivalently

$$
\left|a_{i}+a_{j} z\right|>\frac{|z|^{2}}{1-|z|}
$$

for all $a_{i}, a_{j} \in H$. By continuity, equation (2) holds under small perturbations of $z$. Therefore, there must exist $\delta>0$, such that for all $z^{\prime} \in B(z, \delta)$ we have

$$
\left|a_{i}+a_{j} z^{\prime}\right|>\frac{\left|z^{\prime}\right|^{2}}{1-\left|z^{\prime}\right|} .
$$

Since

$$
\left|\sum_{n=2}^{k} a_{n}\left(z^{\prime}\right)^{n}\right| \leq \frac{\left|z^{\prime}\right|^{2}}{1-\left|z^{\prime}\right|}
$$

for all $\left(a_{n}\right) \in H^{k}$ and $k \in \mathbb{N}$, it follows that $z^{\prime}$ cannot be the zero of a power series or a polynomial. Therefore we must have $R(H) \cap B(z, \delta)=\emptyset$ and $R^{*}(H) \cap B(z, \delta)=\emptyset$.

\section{Proof of Theorem 1.1}

We now turn our attention to proving Theorem 1.1. We start with the following technical proposition.

Proposition 2.1 Let $z \in A_{(1 / 2,1)}$. Suppose $H$ is $2 \cos ^{-1}\left(\frac{5-4|z|^{2}}{4}\right)$-dense, then for any $z^{\prime} \in \overline{B(0,2)}$ there exists $a \in H$ such that $z^{-1}\left(z^{\prime}-a\right) \in \overline{B(0,2)}$.

Remark 2.2 It is useful to point out that the conclusion of this proposition is equivalent to:

$$
\overline{B(0,2)} \subset \bigcup_{a \in H} a+z \overline{B(0,2)}
$$

Proof Let us start by fixing $z^{\prime} \in \overline{B(0,2)}$. Consider the point $z^{\prime} z^{-1}$. Clearly $z^{\prime} z^{-1} \in$ $\overline{B\left(0,2|z|^{-1}\right)}$. Let

$$
S\left(z^{\prime} z^{-1},|z|^{-1}\right):=\left\{\omega \in \mathbb{C}:\left|\omega-z^{\prime} z^{-1}\right|=|z|^{-1}\right\}
$$




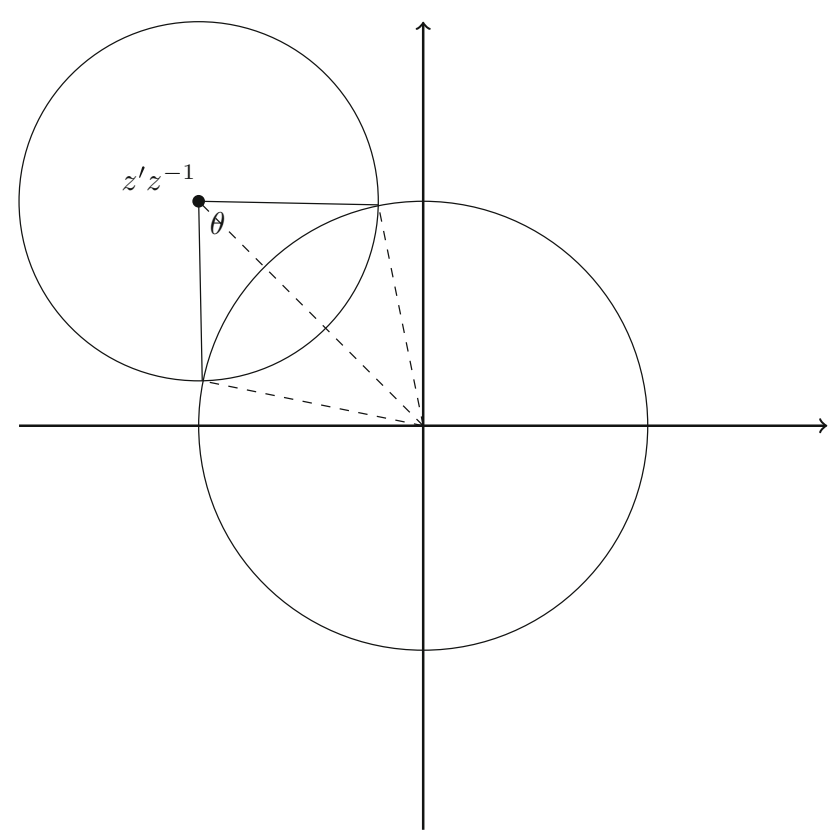

Fig. 1 A diagram of $S_{z^{\prime} z^{-1}}^{\mid z-1}$ intersecting $\overline{B(0,2)}$

be the circle centered at $z^{\prime} z^{-1}$ with radius $|z|^{-1}$.

Since $z \in A_{(1 / 2,1)}$ we must have $S\left(z^{\prime} z^{-1},|z|^{-1}\right) \cap \overline{B(0,2)} \neq \emptyset$. In fact this intersection must contain an arc of $S\left(z^{\prime} z^{-1},|z|^{-1}\right)$. This arc is parameterised by two radii of $S\left(z^{\prime} z^{-1},|z|^{-1}\right)$ with interior angle $\theta$. See Fig. 1 for a diagram describing the intersection of $S\left(z^{\prime} z^{-1},|z|^{-1}\right)$ with $\overline{B(0,2)}$. It is easy to see that the angle $\theta$ is minimised when $z^{\prime} z^{-1}$ is as far from the origin as possible, i.e., when $z^{\prime}$ has modulus 2. Employing elementary techniques from geometry we can see that the angle $\theta$ is at least twice the size of a particular angle of the triangle whose sides have length $|z|^{-1}, 2$, and $2|z|^{-1}$ (see Fig. 1). Therefore, we can use the well-known cosine rule from trigonometry to show that $\theta$ is always bounded below by

$$
2 \cos ^{-1}\left(\frac{5-4|z|^{2}}{4}\right) .
$$

Since $H$ is $2 \cos ^{-1}\left(\frac{5-4|z|^{2}}{4}\right)$-dense as a subset of $S^{1}$, there must exist $a \in H$ such that $z^{\prime} z^{-1}-a z^{-1}$ is contained in the arc of $S\left(z^{\prime} z^{-1},|z|^{-1}\right)$ which intersects $\overline{B(0,2)}$. In particular, for this choice of $a$ we have $z^{-1}\left(z^{\prime}-a\right) \in \overline{B(0,2)}$.

Theorem 1.1 now follows almost immediately from Proposition 2.1.

Proof of Theorem 1.1 By the relations given in Proposition 1.2 to prove Theorem 1.1 it is sufficient to only prove the statement relating to $R^{*}(H)$. Fix $r \in(1 / 2,1)$ and let $H$ be a $2 \cos ^{-1}\left(\frac{5-4 r^{2}}{4}\right)$-dense subset of $S^{1}$. Note that $H$ is automatically $2 \cos ^{-1}\left(\frac{5-4|z|^{2}}{4}\right)$ dense for any $z \in A_{[r, 1)}$. So we can apply Proposition 2.1 for any $z \in A_{[r, 1)}$. Let us 
now fix $z \in A_{[r, 1)}$ and apply Proposition 2.1 when $z^{\prime}=0$. So there exists $a_{0} \in H$ such that $x_{0}:=z^{-1}\left(-a_{0}\right) \in \overline{B(0,2)}$. Rearranging yields

$$
0=a_{0}+x_{0} z
$$

Applying Proposition 2.1 again with $x_{0}$ in the place of $z^{\prime}$ yields $a_{1}$ and $x_{1}:=z^{-1}\left(x_{0}-\right.$ $\left.a_{1}\right)$, such that $x_{1} \in \overline{B(0,2)}$ and

$$
0=a_{0}+a_{1} z+x_{1} z^{2}
$$

One can then apply Proposition 2.1 with $z^{\prime}=x_{1}$. Repeating this procedure indefinitely yields a sequence $\left(a_{n}\right)$ and $\left(x_{n}\right)$ such that $x_{n+1}=z^{-1}\left(x_{n}-a_{n+1}\right)$ for all $n \in \mathbb{N}$. The terms in $\left(x_{n}\right)$ remain in $\overline{B(0,2)}$. Therefore, we are able to repeatedly apply the substitution $x_{n+1}=z^{-1}\left(x_{n}-a_{n+1}\right)$ in (3) and we obtain

$$
0=\sum_{n=0}^{\infty} a_{n} z^{n}
$$

Therefore, $z \in R^{*}(H)$. Since $z$ was arbitrary we have $A_{[r, 1)} \subseteq R^{*}(H)$.

The proof of Theorem 1.1 was based upon ideas from $\beta$-expansions. The argument given relied upon adapting methods from $[2,7]$. The proof can easily be adapted to show that under the hypothesis of the theorem, for every $z^{\prime} \in \overline{B(0,2)}$ there exists $\left(a_{n}\right) \in H^{\mathbb{N}}$ such that $\sum_{n=0}^{\infty} a_{n} z^{n}=z^{\prime}$.

\section{Some Further Problems}

There are some more challenging problems related to root sets $R(H), R^{*}(H)$. We mentioned in the beginning of this paper that there exist various results of multiple roots $[3,4]$. We say that a $z \in \mathbb{C}$ is a multiple root of a holomorphic function $f$ of order $k$ if for all integers $i=0,1,2, \ldots, k$

$$
f^{(i)}(z)=0
$$

Adopting the notation in this paper, we can define for any integer $k \geq 0$ :

$$
\begin{aligned}
R_{k}(H):= & \left\{z \in \mathbb{C}: \exists k>0, \exists\left(a_{n}\right) \in H^{k+1},\right. \\
& \left.P(w)=\sum_{n=0}^{k} a_{n} w^{n}, z \text { is a } k \text {-th order root of } P(w)\right\} .
\end{aligned}
$$




$$
\begin{aligned}
R_{k}^{*}(H):= & \left\{z \in \mathbb{C}: \exists\left(a_{n}\right) \in H^{\mathbb{N}},\right. \\
& \left.P(w)=\sum_{n=0}^{\infty} a_{n} w^{n}, z \text { is a } k \text {-th order root of } P(w)\right\} .
\end{aligned}
$$

Not so much has been studied about the above multiple root set, some partial results can be found in [8]. We can, for example, consider the following questions:

- Are $R_{k}(H), R_{k}^{*}(H)$ dense in any non-degenerate annulus?

- What about the connectedness and path-connectness of $R_{k}(H), R_{k}^{*}(H)$ ?

- What can we say about the boundary of $R_{k}(H), R_{k}^{*}(H)$ ?

Acknowledgements The first author is supported by the EPSRC Grant EP/M001903/1. The second author is supported by a PhD scholarship provided by the School of Mathematics in the University of St Andrews. The authors are grateful to Jonathan Fraser, Tom Kempton, and Sascha Troscheit for fruitful discussions.

Open Access This article is distributed under the terms of the Creative Commons Attribution 4.0 International License (http://creativecommons.org/licenses/by/4.0/), which permits unrestricted use, distribution, and reproduction in any medium, provided you give appropriate credit to the original author(s) and the source, provide a link to the Creative Commons license, and indicate if changes were made.

\section{References}

1. Ahlfors, L.V.: Complex analysis, 3rd edn. McGraw-Hill Book Co., New York (1978)

2. Baker, S.: Generalised golden ratios over integer alphabets, integers, 14 (2014) paper no. A15

3. Beaucoup, F., Borwein, P., Boyd, D.W., Pinner, C.: Multiple roots of $[-1,1]$ power series. J. Lond. Math. Soc. 57(1), 135-147 (1998)

4. Borwein, P., Erdélyi, T., Littmann, F.: Polynomials with coefficients from a finite set. Trans. Amer. Math. Soc. 360, 5145-5154 (2008)

5. Bousch, T.: Connexite locale et par chemins holderiens pour les syst"emes iteres de fonctions. https://pdfs.semanticscholar.org/3a95/0aaad8f0edbcd9efc752fbf153d89002f09e.pdf (1993). Accessed 24 Sept 2017

6. Littlewood, J.E.: On polynomials $\sum^{n} \pm z^{m}, \sum^{n} \pm e^{\alpha_{m} i} z^{m}, z=e^{\theta i}$. J. Lond. Math. Soc. 41, 367-376 (1966)

7. Parry, W.: On the $\beta$ - expansions of real numbers. Acta. Math. Acad. Sci. Hung. 11, 401-416 (1960)

8. Shmerkin, P., Solomyak, B.: Zeros of $\{-1,0,1\}$ power series and connectedness loci for self-affine sets. Exp. Math. 15, 499-511 (2006) 